Classification

Physics Abstracts

$72.80 \mathrm{E}$

\title{
Numerical study of quasiballistic conduction in an InP submicronic layer at $77 \mathrm{~K}$
}

\author{
P. Hesto, J.-F. Pône, M. Mouis and R. Castagné \\ Institut d'Electronique Fondamentale, Bât. 220, Université Paris Sud, 91405 Orsay, France.
}

(Reçu le 6 novembre 1981, accepté le 4 février 1982)

\begin{abstract}
Résumé. - Nous présentons une étude théorique utilisant une simulation de type Monte-Carlo permettant de caractériser les effets balistiques et quasibalistiques dans une structure submicronique en InP à la température de l'azote liquide.
\end{abstract}

Abstract. - A theoretical study based on Monte-Carlo simulation is presented in order to characterize the ballistic or quasiballistic effect in a submicron InP device at liquid nitrogen temperature.

InP is one of the technologically available III. V compound semiconductors the electrical characteristics of which make possible very fast submicron device fabrication. Extensive works $[1,2,3]$ have been published on the non-stationary electron dynamics in InP submicron devices at room temperature. Under such conditions, the number of electron-phonon interactions is large enough so that the momentum relaxation time approximation is valid and then an electron mobility can be defined but the electron transit time being generally of the order of magnitude or shorter than the energy relation time, the overshoot phenomenon must be taken into account.

On the other hand, it is well known that the transport properties of InP are largely improved by lowering the lattice temperature. This is due to the reduction of the electron-phonon interaction probability. At liquid nitrogen temperature, in a submicron device, the number of interactions occurring for one electron during its transit is considerably reduced and ballistic or quasiballistic (i.e. with few interactions) flights must be considered. This paper gives Monte-Carlo simulation results for InP $\mathrm{N}^{+} \mathrm{NN}^{+}$submicron structures showing the relative importance of ballistic and quasiballistic phenomena and their evolution as a function of the bias voltage. The $\mathrm{N}^{+}$contact region has been introduced to take into account the electron injection conditions in the central $\mathrm{N}$ type region [4]. The $\mathrm{N}^{+}$doping level $N_{\mathrm{D}}=9 \times 10^{16} \mathrm{~cm}^{-3}$ was chosen so that the electron concentration $n=5 \times 10^{16} \mathrm{~cm}^{-3}$ was close to the degeneration threshold of the conduction band at $77 \mathrm{~K}$. The $\mathrm{N}^{+}$region thickness $(1000 \AA)$ is quite higher than the Debye length in the mate$\operatorname{rial}\left(L_{\mathrm{D}} \simeq 100 \AA\right)$. The central region thickness $(4000 \AA)$ corresponds to an easily accessible value either by epitaxy technology or in microlithography planar technology, and its doping level has been supposed equal to $10^{14} \mathrm{~cm}^{-3}$, so that the electron-impurity Coulomb interactions can be neglected. The electron-phonon interaction parameters introduced in the Monte-Carlo simulation are taken from the two valley model by Fawcett [5], and the electron impurity Coulomb interactions are treated following Ruch [6]. The electric field in the device is computed using Poisson's equation at each time step (which is equal to $0.01 \mathrm{ps}$ ). 


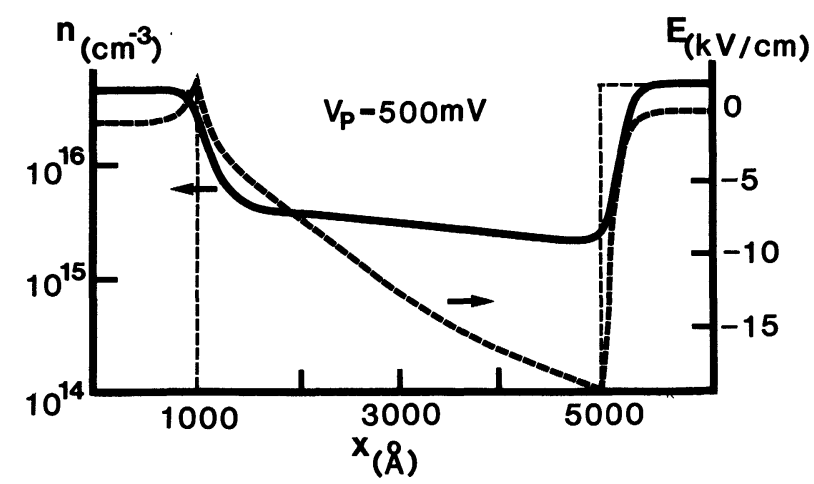

Fig. 1. - Electron concentration (-), ionized impurity concentration (- $\longrightarrow$ ) and electric field (- - - ) distributions along the device for $500 \mathrm{mV}$ bias voltage.

Figure 1 gives the electric field and the electron density distribution in the device for a $500 \mathrm{mV}$ bias voltage. An important electron injection in the central region can be seen due to diffusion from the $\mathrm{N}^{+}$regions in conjunction with the quasi-linear variation of the electric field. The space charge in the $\mathrm{N}$ layer is not analogous to that in a vacuum diode on account of the small proportion of ballistic electrons. In order to obtain the relative importance of ballistic and quasiballistic electrons in the total current, we have determined the electron flux versus the kinetic energy along the field direction for the output electrons. Figure 2 gives this distribution for a $500 \mathrm{mV}$ bias voltage.

Well defined peaks can be seen in this figure. The highest energy peak is due to ballistic electrons. The other peaks are due to electrons which encounter respectively one, two or more interactions in which an optical phonon is emitted and are separated from each other by an energy close to

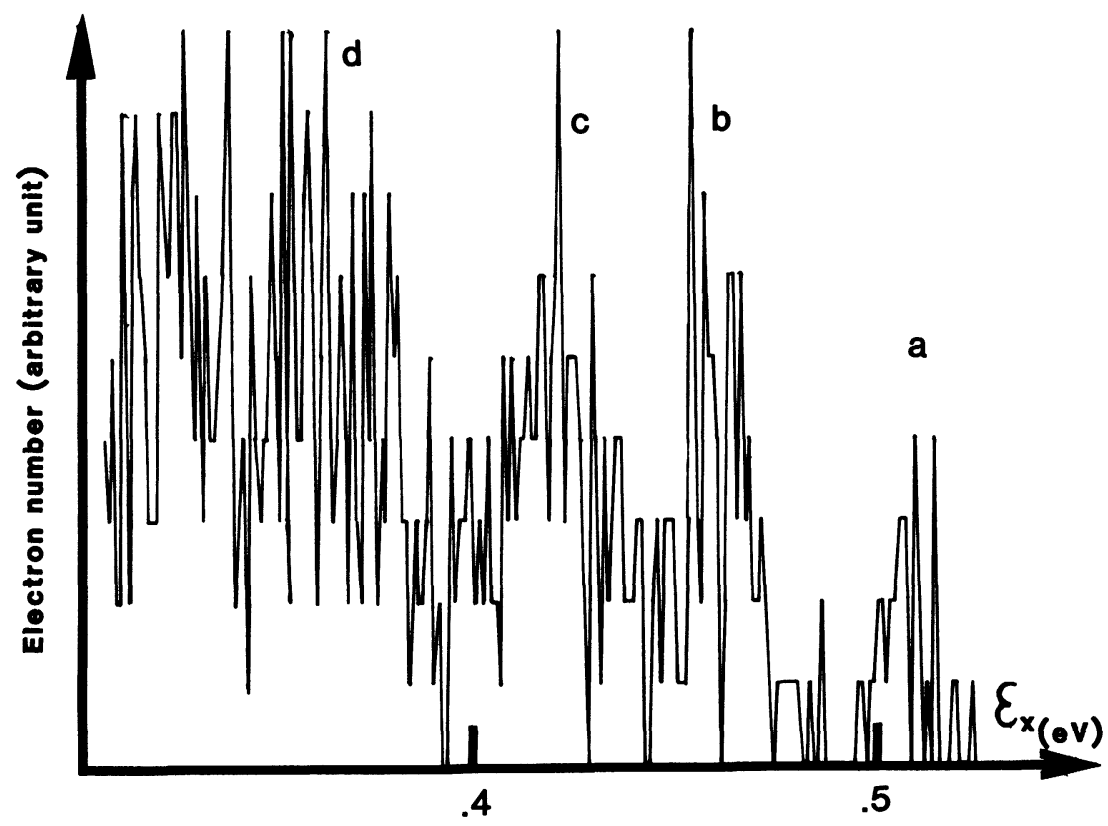

Fig. 2. - Distribution of the electron kinetic energy $\varepsilon_{x}$ along the electric field direction for the output electrons. The potential barrier height of the $\mathrm{N}^{+} \mathrm{N}$ junction is $2 \mathrm{meV}$, the bias voltage being $500 \mathrm{mV}$. 
Table I. - Evolution of the average kinetic energy $\varepsilon_{x}$ and of the contribution of each peak in the energy spectrum, $P$, to the total current with bias voltage. $\phi_{\mathrm{B}}^{*}$ is the potential barrier height of the $\mathrm{N}^{+} \mathrm{N}$ junction.

\begin{tabular}{|c|c|c|c|c|c|c|c|c|c|}
\hline$\underset{(m V)}{V_{p}}$ & $\begin{array}{l}\phi_{\mathrm{B}}^{*} \\
\mathrm{meV}\end{array}$ & $\frac{\text { Peal }}{E_{x_{\text {mov }}}}$ & & Peak & b & Peak & & \multicolumn{2}{|c|}{ Peak d } \\
\hline 50 & 13 & 67 & 5 & & & & & & \\
\hline 100 & 9 & 110 & 1 & 70 & 6 & & & & \\
\hline 200 & 5 & 213 & 1 & 165 & 3 & 125 & 5 & & \\
\hline 300 & 3 & 311 & 1 & 261 & 2 & 223 & 4 & & \\
\hline 400 & 3 & 407 & 1 & 365 & 3 & 319 & 4 & 278 & 6 \\
\hline 500 & 2 & 508 & 1 & 462 & 3 & 419 & 6 & 367 & 7 \\
\hline
\end{tabular}

$43 \mathrm{meV}$, the optical phonon energy. When the total kinetic energy of a $\Gamma$ valley electron stays under $600 \mathrm{meV}$ (which, taking into account the initial electron velocity dispersion, limit bias voltage to about $500 \mathrm{mV}$ ), most of the collisions occur with polar optical phonon emission leading to a small deviation of the electron trajectory. The consequence is a good energy separation between the peaks in the spectrum given in figure 2 with a progressive degradation of this separation as the number of interactions increases. The ballistic peak width corresponds to the initial velocity distribution. Table I shows the evolution with the bias voltage of the contribution of each peak to the total current.

In conclusion, by simulating the conduction in a $\mathrm{N}^{+} \mathrm{NN}^{+}$structure, taking into account the temporal and spatial variations of the electric field due to the electron distribution, it is shown that an important fraction of the current is carried by ballistic or quasiballistic electrons. For a $500 \mathrm{mV}$ bias voltage, $17 \%$ of the total current is due to electrons having at most 3 interactions and flowing through the $\mathrm{N}^{+}$end region with a velocity larger than $1.25 \times 10^{8} \mathrm{~cm} / \mathrm{s}$.

\section{References}

[1] Maloney, T. J., Frey, J., J. Appl. Phys. 48 (1977) 781-787.

[2] Fawcett, W., Hill, G., Electron. Lett. 11 (1975) 80.

[3] Maloney, T. J., Frey, J., Electron. Lett. 10 (1974) 115.

[4] Hesto, P., Pône, J.-F., Castagné, R., in Proceedings of Third International Conference on Hot Carriers in Semiconductors, Montpellier, J. Physique Colloq. 42 (1981) C7-177.

[5] Fawcett, W., Herbert, D. C., J. Phys. C 7 (1974) 1641.

[6] Ruch, J. R., Fawcett, W., J. Appl. Phys. 41 (1970) 3843-3849. 